Peer Reviewed Letter openaccess

\title{
Thermal imaging as a tool in food analysis
}

\author{
Daniela Souza Ferreira \\ Department of Food Technology, Faculty of Food Engineering, University of Campinas (UNICAMP), 13083-862, Campinas, SP, Brazil \\ Contact
}

eng.dsf@gmail.com

https://orcid.org/0000-0002-6359-9503

This Letter reviews the application of thermal imaging for food quality control. Thermal imaging uses a single, portable device, which provides a visible image of the invisible infrared radiation, and can be used for real-time monitoring at low cost compared to spectral imaging technology. To monitor the quality parameters of the sample, classification or regression models are constructed using multivariate analysis or chemometrics tools. Few studies have used thermography for the analysis of food to-date, so this Letter will only address thermal mapping, internal defect detection, adulteration detection and the prediction of compound concentration.

Keywords: infrared thermal image, infrared thermography, food analysis, non-destructive analysis, chemometrics, emissivity

\section{Introduction}

Infrared (IR) thermography, or thermal imaging, was originally developed for military applications, night surveillance and firefighting. The increasing availability of computing technologies and statistical tools has led to wider use of this technology, including in medicine and materials science. ${ }^{1}$ It is a suitable tool for the food industry due to its portability, ability to capture images in real time, non-contact temperature measurement and the ability to allow inspection in inaccessible locations. ${ }^{2}$ Thermal imaging measures the infrared radiation emitted by a surface, resulting in an image related to the temperature distribution and thermodynamic characteristics of each object, including food products. Thus, this technique has the potential for use in food quality control, evaluating the uniformity of heat treatment and the authenticity of food products from image patterns using multivariate analysis. ${ }^{3-5}$ To investigate the application of the technique in food analysis, the ScienceDirect database was searched for the presence of the terms "thermography", "thermal imaging", "thermographic" and "food", resulting in 556 research articles published since 1996. When these terms were searched in the Web of Knowledge database, only 222 articles were found in the field of food science, with the first publication in 1991. ${ }^{6}$ An exponential increase was observed in the annual number of publications since 1991, from one publication to a total of 69 articles in 2019.

\section{Correspondence}

D.S. Ferreira (eng.dsf@gmail.com)

Received: 28 April 2020

Revised: 23 June 2020

Accepted: 13 July 2020

Publication: 21 July 2020

doi: $10.1255 /$ jsi.2020.a7

ISSN: 2040-4565

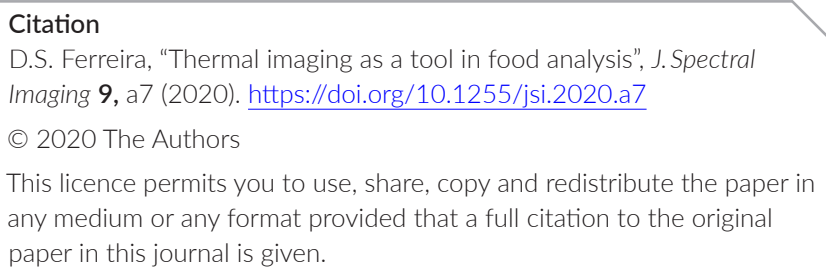

This licence permits you to use, share, copy and redistribute the paper in any medium or any format provided that a full citation to the original paper in this journal is given. 


\section{Theory}

Infrared thermal imaging is an emerging, non-invasive and non-destructive technique, with no contact with the sample and no interference by colour, turbidity or other components from the matrix. Whereas a near infrared (NIR) spectrometer measures the radiation diffusely reflected from the sample, the thermal camera measures the radiation emitted from the object, providing a response on the thermal emissivity and surface temperature distribution of a large area. Thermal IR images, called thermograms, are obtained which are capable of showing temperature gradients of the target, allowing the identification of patterns. The emissivity also depends on the temperature of the material; thus, different temperature distributions of thermal images are obtained for food matrices and chemical or biological materials. ${ }^{4,7}$

The thermography device consists of a portable camera capable of capturing both digital images (RGB images) and thermal images, using an image processing system, which is usually provided by the manufacturer. Its advantages include the low cost and simplicity of the equipment compared to hyperspectral imaging, and operation without the need for fibre optics and a diode array detector. ${ }^{8}$ Thermal imaging cameras usually have a spectral range in the far-infrared between $7.5 \mu \mathrm{m}$ and $13 \mu \mathrm{m}$, produce images of $320 \times 240$ pixels to $1280 \times 960$ pixels and provide high accuracy temperature measurements (up to $1200^{\circ} \mathrm{C}$ ). The camera has IR sensors to detect electromagnetic waves emitted from the surface, which provide a colour response for the temperature distribution over the captured area. Passive or active thermography methods can be used. In passive thermography, there is a difference in temperature between the sample and the background. In active thermography, an energy source is used to produce the thermal contrast between target and background. The contrast can be produced by a laser or flash lamps, which excite the surface with different thermal responses, always at the same distance to control the lighting conditions and to obtain images of a fixed size..$^{3-5,7}$ The choice of the excitation source, the emissivity parameters, as well as the whole theory for calculating the electromagnetic waves emitted by the object have been reported by Doshvarpassand, Wu and Wang. ${ }^{9}$

\section{Applicability}

In food quality control, studies have shown that active thermography (using an energy source) can detect surface defects and internal browning of apples, and the authors reported that such defects were due to the difference in thermal diffusivity rather than emissivity. ${ }^{10,11}$ In this case, a light source was not needed, as opposed to a NIR spectrometer which would be influenced by the apple skin colour. The specific characteristic of this equipment for temperature analysis allows its application for the detection of foreign bodies in food due to the difference in thermal properties, with the use of morphological and statistical analyses (by pattern recognition). The technique enables an effective control of the heat distributed by microwaves during heating, bleaching, drying, pasteurisation and sterilisation processes. ${ }^{6}$ In addition, it allows the identification of corrosion in process lines, even during processing, preventing future contamination. ${ }^{3,4,9,10}$

In food safety, thermal imaging has been used in steam-disinfection of carrots in the process line, with measurements in real time. The uniformity of heat on the surface of the carrot was monitored, and the results showed that treatment did not affect the integrity of the tissues. ${ }^{3}$ Izquierdo et al. ${ }^{5}$ used thermographic imaging to identify honey adulteration with rice syrup in concentrations of $1 \%$, and reported an accuracy of $95 \%$. They were also able to quantify the adulterant with an accuracy of $93 \%$, using Convolutional Neural Network (CNN), a deep learning algorithm (Figure 1). Despite the construction of statistical models, the response of the thermal image is fast and visual, unlike multispectral images.

Oliveira et al. ${ }^{12}$ developed a method to detect alcohol levels in vodka, whisky and cachaça, with an accuracy of 99.4-100.9\%. The method was considered robust, did not need sample preparation and could analyse many samples (480) simultaneously, at reduced analysis time, lower energy consumption, and using green chemistry, since only purified water was used in the microplates to assess the temperature differences of the solutions. Ibarra, Tao and $\mathrm{Xin}^{13}$ used thermal imaging and neural networks to estimate the internal temperature of cooked chicken meat. Unlike other techniques, it was possible to estimate the internal temperature in real time in the process line, without the need to wait a few seconds between one sample and another. 


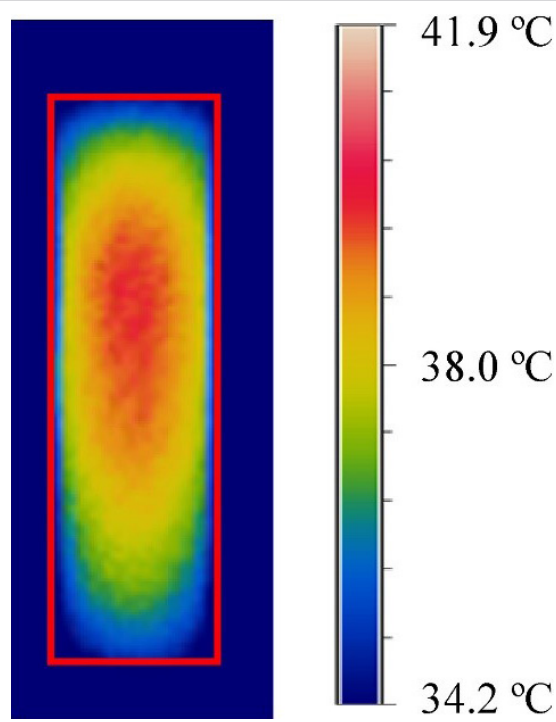

Figure 1. Thermal image (red box) of honey samples. The different temperature distribution of the samples indicates the adulteration detected by $\mathrm{CNN}$ models. ${ }^{5}$ Reproduced from Reference 5 with permission from Elsevier.

\section{Multivariate analysis}

Statistical techniques for image modelling have been applied to improve the use of this technique and to collect information from thermograms, with differentiation between the surface and organic materials. These techniques are called multivariate analyses (chemometrics), which are widely used for classification and quality control, with the advantage of evaluating many samples and variables at the same time. The chemometric models commonly used for classification/differentiation include Partial Least Squares with Discriminant Analysis, Soft Independent Modelling by Class Analogy, Principal Component Analysis, Hierarchical Cluster Analysis and CNN, among others. ${ }^{14-16,5}$

Infrared thermal imaging is an effective alternative for food inspection during processing, mainly due to its low cost and fast identification of organic matter through the classification models.

\section{Conclusion}

This Letter suggests the use of thermal imaging as a viable alternative for food analysis, since it is a low-cost alternative compared to other spectral techniques, uses green chemistry and can be incorporated into portable devices. Applications are still at an early stage, thus further studies are needed for wider industrial application, with emphasis on its potential for real-time evaluation in the process line.

\section{References}

1. F. Amon, A. Hamins, N. Bryner and J. Rowe, "Meaningful performance evaluation conditions for fire service thermal imaging cameras", Fire Safety J. 43(8), 541-550 (2008). https://doi.org/10.1016/j. firesaf.2007.12.006

2. A.A. Gowen, B.K. Tiwari, P.J. Cullen, K. McDonnell and C.P. O'Donnell, "Applications of thermal imaging in food quality and safety assessment", Trends Food Sci. Technol. 21(4), 190-200 (2010). https://doi. org/10.1016/j.tifs.2009.12.002

3. S. Gan-Mor, R. Regev, A. Levi and D. Eshel, "Adapted thermal imaging for the development of postharvest precision steam-disinfection technology for carrots", Postharvest Biol. Technol. 59(3), 265-271 (2011). https://doi.org/10.1016/j.postharvbio.2010.10.003

4. Q. Chen, C. Zhang, J. Zhao and Q. Ouyang, "Recent advances in emerging imaging techniques for nondestructive detection of food quality and safety", Trends Anal. Chem. 52, 261-274 (2013). https://doi. org/10.1016/j.trac.2013.09.007

5. M. Izquierdo, M. Lastra-Mejías, E. GonzálezFlores, J.C. Cancilla, M. Pérez and J.S. Torrecilla, "Convolutional decoding of thermographic images to locate and quantify honey adulteration", Talanta 209(1), 120500 (2020). https://doi.org/10.1016/j. talanta.2019.120500

6. D.L. Goedeken, C.H. Tong and R.R. Lentz, "Design and calibration of a continuous temperature-measurement system in a microwave cavity by infrared imaging", J. Food Proc. Preserv. 15(5), 331-337 (1991). https://doi.org/10.1111/j.1745-4549.1991. tb00176.x

7. A.A. Gowen, B.K. Tiwari, P.J. Cullen, K. McDonnell and C.P. O'Donnell, "Applications of thermal imaging in food quality and safety assessment", Trends Food Sci. Technol. 21(4), 190-200 (2010). https://doi. org/10.1016/j.tifs.2009.12.002

8. L. Senni, P. Burrascano and M. Ricci, "Multispectral laser imaging for advanced food analysis", Infrared Phys. Technol. 77, 179-192 (2016). https://doi. org/10.1016/j.infrared.2016.06.001 
9. S. Doshvarpassand, C. Wu and X. Wang, "An overview of corrosion defect characterization using active infrared thermography", Infrared Phys. Technol. 96, 366-389 (2019). https://doi.org/10.1016/j.infrared.2018.12.006

10. J. Varith, G.M. Hyde, A.L. Baritelle, J.K. Fellman and T. Sattabongkot, "Non-contact bruise detection in apples by thermal imaging", Innov. Food Sci. Emerg. Technol. 4(2), 211-218 (2003). https://doi. org/10.1016/S1466-8564(03)00021-3

11. P. Baranowski, J. Lipecki, W. Mazurek and R.T. Walczak, "Detection of watercore in 'Gloster' apples using thermography", Postharvest Biol. Technol. 47(3), 358-366 (2008). https://doi.org/10.1016/j.postharvbio.2007.07.014

12. A.S. Oliveira, F.M.D. Nora, R.O. Mello, P.A. Mello, B. Tischer, A.B. Costa and J.S. Barin, "One-shot, reagent-free determination of the alcoholic content of distilled beverages by thermal infrared enthalpimetry", Talanta 171(15), 335-340 (2017). https://doi. org/10.1016/j.talanta.2017.05.011

13. J.G. Ibarra, Y. Tao and H. Xin, "Combined IR imaging-neural network method for the estimation of internal temperature in cooked chicken meat", Optical Eng. 39(11), 3032-3038 (2000). https://doi. org/10.1117/1.1314595

14. P.A. Lachenbruch, Discriminant Analysis. Hafner Press, New York (1975).

15. S. Wold, "Pattern recognition by means of disjoint principal components models", Pattern Recogn. 8(3), 127-139 (1976). https://doi.org/10.1016/00313203(76)90014-5

16. M.M.C. Ferreira, "Preparação dos dados para análise", in Quimiometria-Conceitos, Métodos e Aplicações, Ed By M.M.C. Ferreira. Editora da Unicamp, Campinas, SP, 496, pp. 29-105 (2015). 